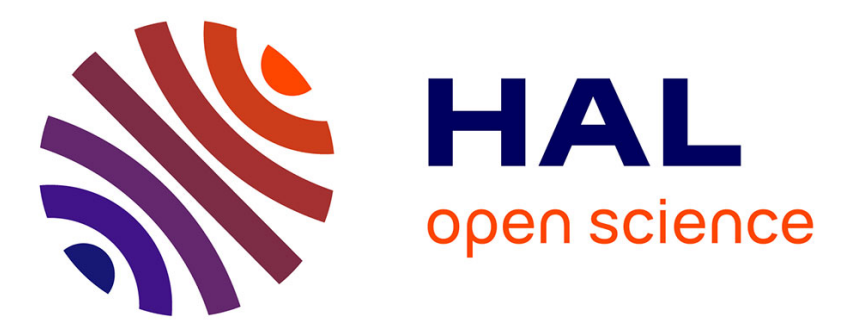

\title{
The focusing problem for the Leith model of turbulence: a self-similar solution of the third kind
}

\author{
S. Nazarenko, V N Grebenev, S. Medvedev, Sébastien Galtier
}

\section{To cite this version:}

S. Nazarenko, V N Grebenev, S. Medvedev, Sébastien Galtier. The focusing problem for the Leith model of turbulence: a self-similar solution of the third kind. Journal of Physics A: Mathematical and Theoretical, 2019, 52 (15), pp.155501. 10.1088/1751-8121/ab0da5 . hal-02169311

\section{HAL Id: hal-02169311 \\ https://hal.sorbonne-universite.fr/hal-02169311}

Submitted on 1 Jul 2019

HAL is a multi-disciplinary open access archive for the deposit and dissemination of scientific research documents, whether they are published or not. The documents may come from teaching and research institutions in France or abroad, or from public or private research centers.
L'archive ouverte pluridisciplinaire HAL, est destinée au dépôt et à la diffusion de documents scientifiques de niveau recherche, publiés ou non, émanant des établissements d'enseignement et de recherche français ou étrangers, des laboratoires publics ou privés. 


\title{
The focusing problem for the Leith model of turbulence: a self-similar solution of the third kind
}

\author{
S. V. Nazarenko ${ }^{1}$, V. N. Grebenev ${ }^{2} \ddagger$ S. B. Medvedev ${ }^{3}$ and S. \\ Galtier $^{4,5}$ \\ ${ }^{1}$ Institute de Physique de Nice, Universite Côte D'Azur, Ave. Joseph Vallot, Nice \\ 06100, France \\ ${ }^{2}$ Faculty of Mathematics and Statistics, Ton Duc Thang University, Ho Chi Minh \\ City, Vietnam \\ ${ }^{3}$ Institute of Computational Technologies SD RAS, Lavrentiev avenue 6, Novosibirsk \\ 630090, Russia \\ ${ }^{4}$ Laboratoire de Physique des Plasmas, École polytechnique F-91128 Palaiseau \\ Cedex, France \\ ${ }^{5}$ Univ. Paris-Sud, Observatoire de Paris, Univ. Paris-Saclay, CNRS, Sorbonne \\ Univ., Institut universitaire de France
}

\begin{abstract}
Time-dependend evolution of hydrodynamic turbulence corresponding to formation of a thermodynamic state at the large-scale part of the spectrum is studied using the inviscid Leith model. In the wave vector space, the evolution leads to shrinking of the zero-spectrum "hole" - the so-called focusing problem. However, in contrast with the typical focusing problem in the nonlinear filtration theory, the focusing time is infinite for the Leith model. Respectively, the evolution is described by a self-similar solution of the third kind (discovered in Nazarenko, Grebenev [Phys A: Math. Theor. 50, 3, 2017]), and not the second kind as in the case of the typical filtration problem. Using a phase-plane analysis applied to the dynamical system generated by this type of similarity, we prove the existence of a new self-similar spectrum to this problem. We show that the final stationary spectrum scales as the thermodynamic energy equipartition spectrum, $E \sim k^{2}$.
\end{abstract}

PACS numbers: 47.27.Eq, 47.27.ed, 02.60Lj

$\ddagger$ Corresponding author, e-mail: vladimirgrebenev@tdt.edu.vn 


\section{Introduction}

The Leith model of turbulence [1] was extensively studied in [2]-[4] and [5]. This model is represented by a nonlinear diffusion equation in which the energy spectrum $E(k, t)$ satisfies the equation

$$
\frac{\partial E}{\partial t}=\frac{1}{8} \frac{\partial}{\partial k}\left(k^{11 / 2} E^{1 / 2} \frac{\partial}{\partial k}\left(E / k^{2}\right)\right)-\nu k^{2} E,
$$

where $t$ is time, $k$ is the absolute value of the wavenumber and $\nu$ is the kinematic viscosity coefficient. For more details about the physical background, see [6], [7]. Discussion of the Leith model in the context of other turbulence closures can be found in [8].

The energy flux in the Leith model is defined as

$$
\epsilon(k, t)=-\frac{1}{8} k^{11 / 2} E^{1 / 2} \frac{\partial}{\partial k}\left(E / k^{2}\right) .
$$

Notice that in the inertial range, when the viscosity term can be neglected, equation (1) admits two fundamental stationary scaling solutions: the thermodynamic spectrum $E(k) \sim k^{2}$ corresponding to zero flux, $\epsilon \equiv 0$, and the Kolmogorov spectrum $E(k) \sim k^{-5 / 3}$ corresponding to a constant flux, $\epsilon=$ const $>0$.

In [2], we studied transient solutions for the Leith model arising from an initial spectrum compactly supported at low $k$-wavenumbers. It was shown that the evolution becomes self-similar just before breaking of energy conservation at some finite time $t=t_{*}$ at which the front of the spectrum reaches $k=\infty$. This is the so-called

self-similarity of the second kind [9] at pre- $t_{*}$ stage of the spectrum evolution. This regime does not exhibit the scaling inherited from the Kolmogorov spectrum. Namely, the transient spectrum behind the propagating front was found and asymptotes to a power-law spectrum $E \sim k^{-x^{*}}$ with $x^{*}$ which is greater than the Kolmogorov exponent, $x^{*} \approx 1.85>5 / 3$. Previously, similar behaviour of a transient spectrum exhibiting an anomalously steep power law was found numerically in MHD wave turbulence [10], [11].

We remind that, according to the Zeldovich-Raizer classification [9], a self-similar solution is of the first kind if its similarity coefficients ( $a$ and $b$ in the text below) are completely determined by a conservation law. A self-similar solution is of the second kind if its similarity coefficients can only be found by solving a nonlinear eigenvalue problem. There is also a third possibility when the similarity coefficients are completely determined by a scaling inherited from the preceding self-similar stage; this is the third kind self-similarity (see below).

A more general Lie point symmetry analysis had been performed in [3] for both the viscous and the inviscid versions of equation (1). We gave a complete group classification of all invariant solutions of these equations.

In [4], we presented a comprehensive study and full classification of the stationary solutions in Leith's model with a generalised viscosity. The solutions obtained were interpreted in terms of their physical meanings as low and high Reynolds number direct and inverse energy cascades. These include the Kolmogorov, the thermodynamic and the 
mixed solutions which arise in this model with various types of the boundary conditions as $t \rightarrow \infty$.

The question about the evolution in Leith's model for $t>t_{*}$ was investigated in [5] and this is the so-called post- $t_{*}$ stage of energy spectrum $E(k, t)$. Numerical simulations presented in [6] demonstrate that during this period of time there is a reflected wave propagating from large to small $k$ which replaces the steep power-law with exponent $x^{*}$ with a shallower spectrum whose exponent is Kolmogorov's $5 / 3$. It was shown that this post- $t_{*}$ stage is described by a self-similar solution of the inviscid Leith equation (1) of the third kind [5]. Nonstationary solutions corresponding to "warm cascades" [6] characterized by a thermalised spectrum at large wavenumbers were also observed. Transition from the Kolmogorov to the thermalised range is characterized by the presence of a range with a depleted spectrum. The depletion on the spectrum is especially pronounced in the case of strong reflections which occur for very abrupt dissipation functions at high wave numbers, e.g. hyperviscosity. A similar effect was observed in the numerical simulations of the Fourier-truncated Euler equation in [12]. They called such a spectrum depletion a "secondary dissipation".

The previous studies of the evolving spectrum mostly concentrated on the range of wave numbers greater than the initial wave numbers, $k>k_{0}$. This is the range where the turbulent cascade occurs and the Kolmogorov spectrum forms. In the present paper, we consider the range of small wave numbers, $k<k_{0}$, for which we expect evolution towards a thermodynamic steady state with equipartition of energy. Such an evolution corresponds to the so-called focusing problem: the spectral front propagation from the initial to the small wavenumbers thereby filling the "hole" centered at $k=0$. In section 2, we consider the focusing problem for the inviscid Leith model and overview the focusing problem settings. In section 3 , we show that the self-similar ansatz of the second kind for the inviscid Leith equation is broken. Therefore the focusing time $S$ is infinite for this model. It means that the evolution corresponds to similarity of the third kind which is studied in section 4 . We do it by analysing the behavior of trajectories of the dynamical system derived and demonstrate the existence of a trajectory which corresponds to a solution of the focusing problem. The results of numerical simulations of the forceddissipated Leith model which conform stability of the analytical self-similar solution obtained and the fact that it is reached asymptotically for large time are presented in section 5 .

\section{The focusing problem: overview of the problem settings}

We consider the inviscid Leith model,

$$
\frac{\partial E}{\partial t}=\frac{1}{8} \frac{\partial}{\partial k}\left(k^{11 / 2} E^{1 / 2} \frac{\partial}{\partial k}\left(E / k^{2}\right)\right) .
$$

In the focusing problem, it is assumed that initially at time $t=t_{0}$ there is a nonempty compact set $D=\{[0, d] \subset \mathbb{R}\}$ such that the spectrum $E\left(k, t_{0}\right) \equiv 0$ and $E\left(k, t_{0}\right)>0$ for a non-zero measure subset of $\mathbb{R} \backslash D$. As the time evolves, the spectrum front will enter 
$D$ and eventually reach all points of $D \backslash 0$ at some non-zero time $S$. We will call $S$ the focusing time; it can be finite or infinite. Of considerable interest here is the behavior of $E(k, t)$ near the focusing time $S$, especially its asymptotics at $k \rightarrow 0$ which often takes form of a power law. This formulation is following a general approach in the theory of the porous medium equations [13].

Typically, the focusing solution of the porous medium equation

$$
\frac{\partial u}{\partial t}=\Delta u^{m}
$$

is a self-similar solution of the second kind for the "slow" diffusion case $m>1$. There is quite a different situation for the linear diffusion case $m=1$, and the "fast" diffusion case $m<1$ [13]. The focusing time is zero for these cases and the self-similar ansatz is broken.

In turbulence, the focusing problem can be associated with studying a front propagation from large to small wavenumbers $k$. We will be concerned with the case where energy is continuously pumped into the system at some high wavenumbers around $k_{0}$ and dissipated by viscosity at even higher wave numbers $k_{d} \gg k_{0}$ so that a steady state is eventually forming which is, according to the results of [4], with the thermodynamic spectrum $E_{s}(k) \sim k^{2}$ at $k \rightarrow 0$. Thus we impose a condition that

$$
E(k, t) \rightarrow k^{2}, \quad \text { as } t \rightarrow S, \quad k \rightarrow 0 .
$$

Note that this formulation is very general, because choosing a different constant prefactor in (5) can be considered by a simple rescaling of the solution.

\section{Self-similar ansatz of the second kind is broken}

A priory, it is not clear if the focusing time $S$ is finite, zero or infinite and, therefore, it is not clear what kind of the self-similarity is relevant. Let us first consider a possibility that $S$ is finite and consider the self-similar anzatz of the second kind. Thus, we will seek solutions of equation (3) in the following form,

$$
E=(S-t)^{a} F(\eta), \quad \eta=k / k_{*}, \quad k_{*}=(S-t)^{b},
$$

where $a$ and $b$ are constants satisfying the dimensional condition

$$
a=-2-3 b .
$$

Equation for $F(\eta)$ reads:

$$
-a F+b \eta \frac{d F}{d \eta}=\frac{1}{8} \frac{d}{d \eta}\left(\eta^{11 / 2} F^{1 / 2} \frac{d}{d \eta}\left(\eta^{-2} F\right)\right) .
$$

According to the focusing problem setting, we look for a solution of (8) such that

$$
F(\eta) \equiv 0 \quad \text { for } \quad \eta \leq 1 .
$$

Note that setting the hole boundary to be at $\eta=1$ does not restrict generality, because any other value can be considered by rescaling of the considered problem. We are interested in solutions with the boundary $k_{*}(t)$ moving toward smaller wave numbers, 
thus $b>0$. We also impose the condition that the shape of $F(\eta)$ at $\eta \rightarrow \infty$ must correspond to a stationary (time-independent) spectrum $E(k)=k^{2}$. This fixes $a / b=2$. But together with (7), this condition is incompatible with $b>0$. Thus the self-similar ansatz of the second kind is broken, i.e. there exist no self-similar solution of the second kind corresponding to the focusing problem where the stationary thermodynamic spectrum $E(k)=k^{2}$ forms in a finite time.

\section{Infinite-time focusing: self-similar solution of the third kind}

Taking into account that the self-similar ansatz (6) is broken, we will look for a solution to the focusing problem in the following self-similar form

$$
E(k, t)=t^{a} F(\eta), \quad \eta=k t^{b} .
$$

With this ansatz, the self-consistency condition takes the form

$$
a=-2+3 b \text {. }
$$

We consider

$$
a=-\frac{2 x}{x-3} \quad \text { and } \quad b=\frac{-2}{x-3}
$$

with $x<0$ and therefore the self-consistency condition (11) holds. Then the equation for $F(\eta)$ reads:

$$
-\frac{2}{x-3}\left(\eta \frac{d F}{d \eta}+x F\right)=\frac{1}{8} \frac{d}{d \eta}\left(\eta^{11 / 2} F^{1 / 2} \frac{d}{d \eta}\left(\eta^{-2} F\right)\right) .
$$

We complement this equation by three conditions corresponding to the focusing setup: (i) $F(\eta) \equiv 0$ for $0 \leq \eta \leq 1$. (ii) We require that the flux of energy $\epsilon$ turns into zero at the points where $\eta=0$. This rules out nonphysical solutions with a finite sink moving in the wavenumber space. (iii) $F(\eta)$ at $\eta \rightarrow \infty$ is such that the spectrum $E(k)$ is time independent, specifically, corresponds to the stationary (time-independent) spectrum $E(k)=k^{2}$ i.e. $F(\eta) \sim \eta^{2}$ for $\eta \gg 1$. We emphasize that the stationarity of spectrum $E(k)=k^{2}$ implies that our turbulence is continuously forced. The fact that the forcing wavenumber $k_{0}$ is fixed means that the corresponding value of the similarity variable tends to infinity, $\eta_{0}(t) \rightarrow \infty$. Hence there is a range of the self-similar solution which is simultaneously far from the front and from the forcing scale, $1 \ll \eta \ll \eta_{0}$, and this is the range where we impose the stationary thermodynamic spectrum.

With $a=3 b-2$ we have a one-parametric family of self-similar solutions,

$$
E(k, t)=t^{3 b-2} F\left(k t^{b}\right) .
$$

The value of parameter $b$ is to determined by the asymptotic of $F(\eta)$ as $\eta \rightarrow \infty$ that corresponds to a time independent spectrum. Therefore, the above-mentioned focusing setup leads to a self-similarity of the third kind i.e. the similarity coefficients $a$ and $b$ are not determined by dimensional analysis of the equation under consideration or conservation laws but rather from the post self-similar stage as here. With this, the 
self-similar exponent $b$ has to be equal to $2 / 5$ to satisfy the condition (iii). Interestingly, this choice of $b$ coincides with the Saffman similarity law [14].

The easiest way to understand the main features of the self-similar ansatz (10) is to consider the phase space plot of the respective dynamical system associated with equation (13). Equation (13) can be transformed into an autonomous system by the same substitutions as in [5]. The resulting autonomous dynamical system is:

$$
\begin{aligned}
& \frac{d \rho}{d \tau}=\frac{3}{2} \rho(\rho+\sigma), \\
& \frac{d \sigma}{d \tau}=\frac{1}{3}\left(5 \rho^{2}+6 \rho \sigma-9 \sigma^{2}-\frac{10}{x-3}(3 \sigma+x \rho)\right)
\end{aligned}
$$

where $x=a / b$ and equals -2 for $b=2 / 5$. Here we use the notations

$$
\rho(\tau)=f(s), \quad \sigma(\tau)=g(s), \quad s=\ln \eta, \quad d s=f(s) d \tau
$$

and

$$
F=\frac{1}{25} \eta^{-3} f^{2}, \quad \frac{d F}{d \eta}=\frac{3}{25} \eta^{-4} f g .
$$

The phase portrait of this system for $x=-2$ is shown in Figures 1 .

Fixed points of the system (15), (16) in the semi-plane $\rho \geq 0$ for $x=-2$ are

$$
P 1=\left(\rho_{1}, \sigma_{1}\right)=(0,0) \quad \text { and } \quad P 2=\left(\rho_{2}, \sigma_{2}\right)=\left(0, \frac{2}{3}\right) .
$$

The classification of $P 1$ and $P 2$ was done in [5]. Namely, $P 1$ is an unstable saddle-node with its stable manifold along the $\sigma$-axis, the fixed point $P 2$ is a saddle with its unstable manifold along the $\sigma$-axis. A heretoclinic orbit $H$ connects $P 1$ and $P 2$ along the $\sigma$-axis. The slow (central) manifold of the equilibrium of $P 1$ is directed into the first quadrant with the slope $2 / 3$. More specifically, $\sigma=(2 / 3) \rho$ and the dynamics of $\rho(\tau)$ on this central manifold is governed by the equation

$$
\frac{d \rho}{d \tau}=\frac{5}{2} \rho^{2}
$$

which can be easily integrated:

$$
\rho(\tau)=\frac{1}{C-\frac{5}{2} \tau}
$$

where $C$ is a positive constant. On this orbit, denoted by $U 1$, we have:

$$
\rho(\tau) \sim \frac{1}{C-\frac{5}{2} \tau}, \quad \sigma(\tau) \sim \frac{3}{2 C-5 \tau}
$$

as $\tau \rightarrow-\infty$. It corresponds to the power law scaling

$$
F(\eta) \sim \eta^{2}
$$

This is the same as it has been done in [2], see the formula (11) there. Notice that $F(\eta)=\eta^{2}$ is an exact solution of equation (13) corresponding to the stationary thermodynamic spectrum $E \sim k^{2}$. However, this solution does not satisfy the condition (i) in the left boundary and, therefore, is not the self-similar solution we are looking for. 
Near the fixed point $P 2$, along its stable manifold $U 2$, we have

$$
\rho \sim \exp (\tau+C), \quad \sigma \sim 2 / 3
$$

as $\tau \rightarrow-\infty$. Orbit $U 2$ which expands into the first quadrant. It corresponds to the scaling

$$
F(\eta) \sim(\eta-1)^{2}
$$

for $\eta-1 \ll 1$ or $\tau \rightarrow-\infty$. With the formulas (18) and (24), we get that $F(\eta)$ together with $d F(\eta) / d \eta$ vanishes at $\eta=1$. Further and similar to [6], the formula (25) follows by expanding $F(\eta)$ into the Taylor series with respect to a small $\eta-1$ taking into account equation (13). In the leading order it gives exactly (25). Let us consider the orbit $U_{f}=U 2 \cup H$. With the inverse time $\hat{\tau}=-\tau$, the orbit $U 2$ will reach small values of $\rho$ such that $\rho \ll 1$ and $\rho \ll \sigma$ and terminates at $P 2$. With this, $\rho \rightarrow 0$, $\eta \rightarrow 1$ as $\hat{\tau} \rightarrow \infty$ and $F(\eta) \sim(\eta-1)^{2}, \eta-1 \ll 1$. Further, the dynamics is determined by moving along the heteroclinic orbit $H$ towards $P 1$. The motion along $H$-orbit gives $F(\eta) \equiv 0$ for $\eta \leq 1$. Thus we see that the $U_{f}$-orbit looks like a suitable solution to the focusing problem for equation (13). It only remains to verify the conditions (ii) and (iii) from the focusing setup. For this, we investigate the behavior of $U 2$-orbit as $\tau \rightarrow \infty$. We notice that the thermodynamic line $U 2$ is

$$
\sigma=-\frac{x}{3} \rho \equiv \frac{2}{3} \rho, \quad \text { for } x=-2 .
$$

It is the phase-plane realization of the thermodynamic spectrum $E(k)=k^{2}$ and the function (26) is an exact solution of the dynamical system (15). It is easy to see that the unstable orbit $U 2$ goes from equilibrium $P 2$ to infinity and asymptotes the thermodynamic line. Indeed, $U_{x}$-orbit cannot asymptote to neither the $\sigma$-axis because, according to the first equation of $(15), \rho(\tau)$ is a non-decreasing function. Also, orbit $U 2$ does not asymptote to the $\rho$-axis because it lies above $U 1$. Further, orbit $U 2$ does not asymptote to a line $\rho=$ const $>0$ or a line $\sigma=$ const $>0$ as it would contradict the first equation of (15). Therefore, the $U 2$-orbit goes to infinity in both $\rho$ and $\sigma$ by the Poincaré-Bendixon theorem. Further, for $\rho \gg 1$ and $\sigma \gg 1$ the dynamics of $U 2$-orbit in the leading order is governed by

$$
\begin{aligned}
& \frac{d \rho}{d \tau}=\frac{3}{2} \rho(\rho+\sigma), \\
& \frac{d \sigma}{d \tau}=\frac{1}{3}\left(5 \rho^{2}+6 \rho \sigma-9 \sigma^{2}\right) .
\end{aligned}
$$

The system (27) corresponds exactly to the thermodynamic line. Therefore, $U 2$-orbit asymptotes the thermodynamic line. With this, we obtain $F \sim \eta^{2}$ as $\eta \rightarrow \infty$ which is the condition (iii).

Consider the spectrum $E(k, t)=t^{-4 / 5} F\left(k t^{2 / 5}\right)$. The final profile is easily calculated. For a fixed $k$ we have $\eta=k t^{2 / 5} \rightarrow \infty$ as $t \rightarrow \infty$ and

$$
E(k, t) \rightarrow k^{2} \text {. }
$$

The convergence of $E(k, t)$ follows from the monotone convergence of $F(\eta) \rightarrow \eta^{2}$ as $\eta \rightarrow \infty$. Function $k_{0}(t)=t^{-2 / 5}$ represents a sharp left boundary of the support of 


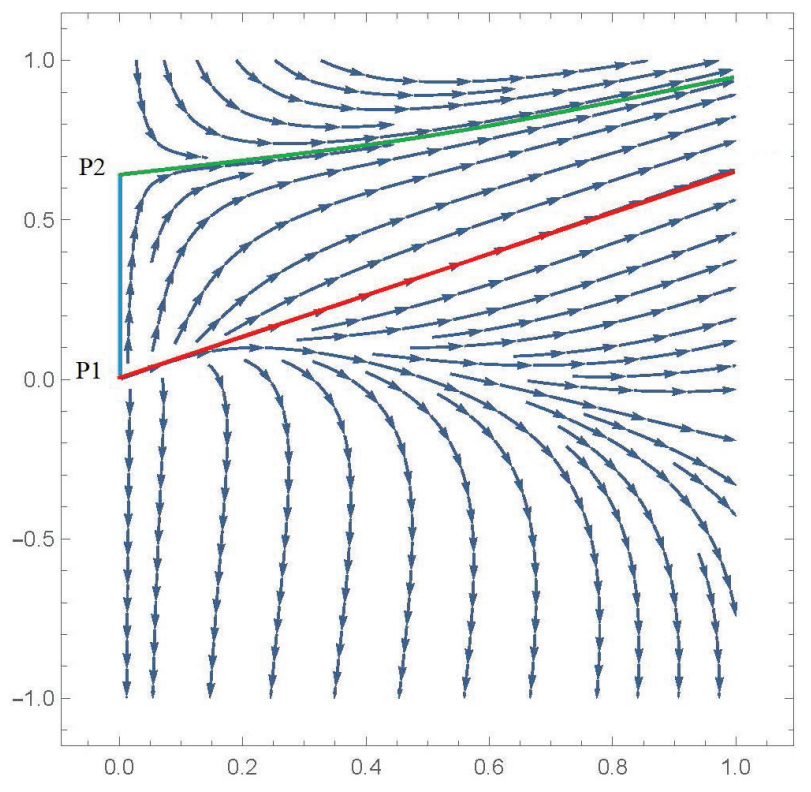

Figure 1. Phase portrait of the dynamical system for $x=-2$ plotted on the $(\rho, \sigma)$ plane. The thermodynamic line is shown by red line. The separatrix $U 2$ is shown in green and the heteroclinic orbit $H$ is the blue line.

$E(k, t)$ corresponding to $\eta=1$. We get that $k_{0}(t) \rightarrow 0$ as $t \rightarrow \infty$ i.e. shrinking of the zero-spectrum "hole" of the energy spectrum $E(k, t)$ occurs in an infinite time.

It is easy to see that the $U_{f}$-orbit is different from the other orbits of the dynamical system (15),(16) in that it represent a unique solution to the focusing problem. Indeed, orbits below the $U 2$-separatrix are connected to $P 1$ moving backward in time along the central (slow) manifold $\sigma=(2 / 3) \rho+O(\rho)$. With this, the behavior of $\rho$ and $\sigma$ is described by the formulas (22) which leads to the power scaling $F \sim \eta^{2}$ for $\eta \ll 1$. This behavior of $F(\eta)$ is ruled out in the focusing problem. Orbits of the phase space above the $U 2$-separatix correspond to the inverse-cascade solutions: they end at a finite wave number (front) with an infinite negative derivative, such that the energy flux experiences a finite jump. This would imply the presence of a finite time-dependent energy sink at the moving front, which is nonphysical. Indeed, at $\rho \rightarrow 0$ and $\sigma \rightarrow \infty$ these orbits asymptotically behave as $F=C \eta^{-3}(\ln \eta)^{2 / 3}, \eta \rightarrow 1$, where $C=$ const $>0$, and for the derivative $d F / d \eta$ we have $d F / d \eta=(2 C / 3) \eta^{-4}(\ln \eta)^{-1 / 3}$ at $\eta \rightarrow 1$. The energy dissipation in the similarity variable $\eta$ reads

$$
\epsilon=-\frac{t^{-11 / 5}}{8}\left(-2 \eta^{5 / 2} F^{3 / 2}+\eta^{15 / 2} F^{1 / 2} \frac{d F}{d \eta}\right) .
$$

Substituting the above-mentioned formulas for $F$ and $d F / d \eta$ into (29), we get that the energy flux actually experience a finite jump at the front: $\left.\epsilon\right|_{\eta=1}=-\frac{C^{3 / 2} t^{-11 / 5}}{12}$. Thus, the only correct relevant solution of the focusing problem corresponds to the $U_{f}$-orbit.

Summarising the results above, we get:

Theorem 4.1 There exists a unique solution to the focusing problem for the equation (3) having the self-similar form the third-kind in the form (10) with boundary conditions 
(i)-(iii). In terms of the orbits of the dynamical system (15),(16), this solution corresponds to the orbit $U_{f}=U 2 \cup H$. The interface $k_{0}(t)$ of the self-similar energy spectrum $E(k, t)$ behaves as $k_{0}(t) \sim t^{-2 / 5}$ so that the hole of $E(k, t)$ is filled in infinite time with $E(k, t) \rightarrow k^{2}$ at any fixed $k$ as $t \rightarrow \infty$.

\section{Numerical simulations}

A priori, there is no guaranty that the found self-similar solution is stable and that it is reached asymptotically for large time when an initial value problem is considered. Establishing these properties analytically is a hard task. However, numerical simulations could provide us with a reliable tool to study stability and asymptotic behaviour.

We have simulated numerically the Leith model (1) in which we have included a forcing term so that the system has a non-zero steady state,

$$
\frac{\partial E}{\partial t}=\frac{\partial}{\partial k}\left(k^{11 / 2} \sqrt{E} \frac{\partial}{\partial k}\left(\frac{E}{k^{2}}\right)\right)-\nu k^{2} E+f,
$$

where $\nu=4 \times 10^{-5}$ and $f \equiv f(k)$ is a time-independent forcing. A logarithmic subdivision of the $k$-axis is used with $k_{i}=2^{i / 10}$ and $i$ an integer varying between 0 and 249. The forcing excites modes $i=[119-11,119+11]$ which corresponds to forcing wavenumbers $k_{f} \in[1783,8192]$. The forcing is $f(k) \sim \exp \left(-k_{i}^{2} / k_{119}^{2}\right)$ for $i \geq 119$ and $f(k) \sim \exp \left(-k_{119}^{2} / k_{i}^{2}\right)$ for $i \leq 119$. A Crank-Nicholson numerical scheme is implemented for the nonlinear term and an adaptive time-step is used [16].

Figure 2 shows the time evolution (every 2000 time-steps) of the energy spectrum. We clearly see the shape of the forcing because it gives its imprint to the initial spectrum (in magenta). The Kolmogorov spectrum $k^{-5 / 3}$ is quickly formed (it appears at $t=2 \times 10^{-6}$ ) while the $k^{2}$ scaling needs much longer time to develop: at the final time of the simulation, $t=2 \times 10^{-3}$, the thermodynamics solution spans over a decade but it still continues to extend toward the smallest wavenumbers. Note that when the Kolmogorov spectrum is formed the energy of the system is around $\int E(k) d k \sim 3000$

and at the end of the simulation it is around 7000, which means that large portion of the energy is accumulating in the thermodynamic part.

Figure 3 shows the temporal evolution of the spectral energy flux $\epsilon(t, k)$ during the same time period as for the energy spectrum shown in Figure 2. We clearly see that the Kolmogorov spectrum corresponds to a constant positive flux $\left(\epsilon \sim 4 \times 10^{9}\right)$. Interestingly, this plot reveals that the plateau is formed at $t=3 \times 10^{-6}$, which is slightly later than the time needed to form the $k^{-5 / 3}$ spectrum. As expected, the thermodynamics solution corresponds to a wavenumber range with nearly zero flux (we find $\epsilon \sim 10^{-43}$ ).

As we see, the thermodynamic spectrum does form in the forced-dissipated Leith model in the range of small wavenumbers at large times and, therefore, the found analytical self-similar solution is stable and provides correct large- $t$, small- $k$ asymptotics for the initial value problem. This inviscid solution appears to be insensitive to the presence of viscosity if $\nu$ is small enough so that the viscous effects are strong at high wavenumbers only. 


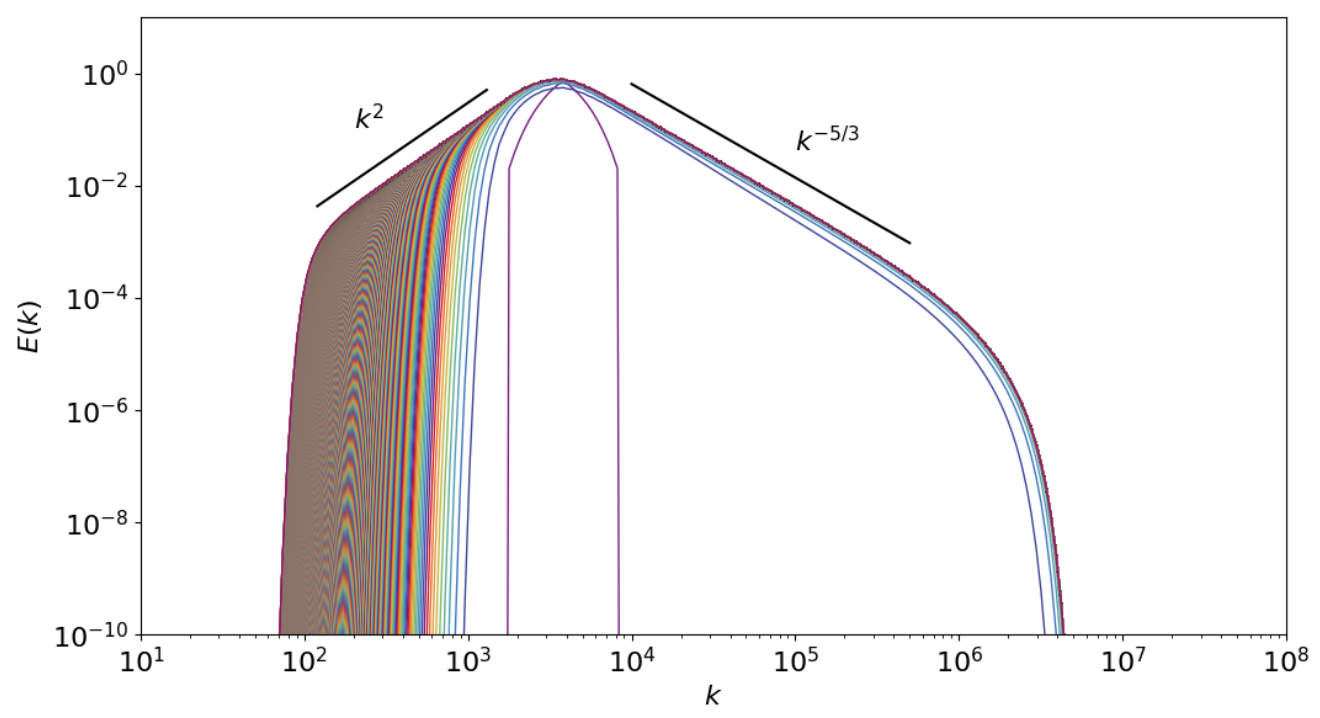

Figure 2. Time evolution of the energy spectrum $E(k, t)$. The Kolmogorov $k^{-5 / 3}$ spectrum is quickly formed while the large-scale $k^{2}$ scaling needs a much longer time.

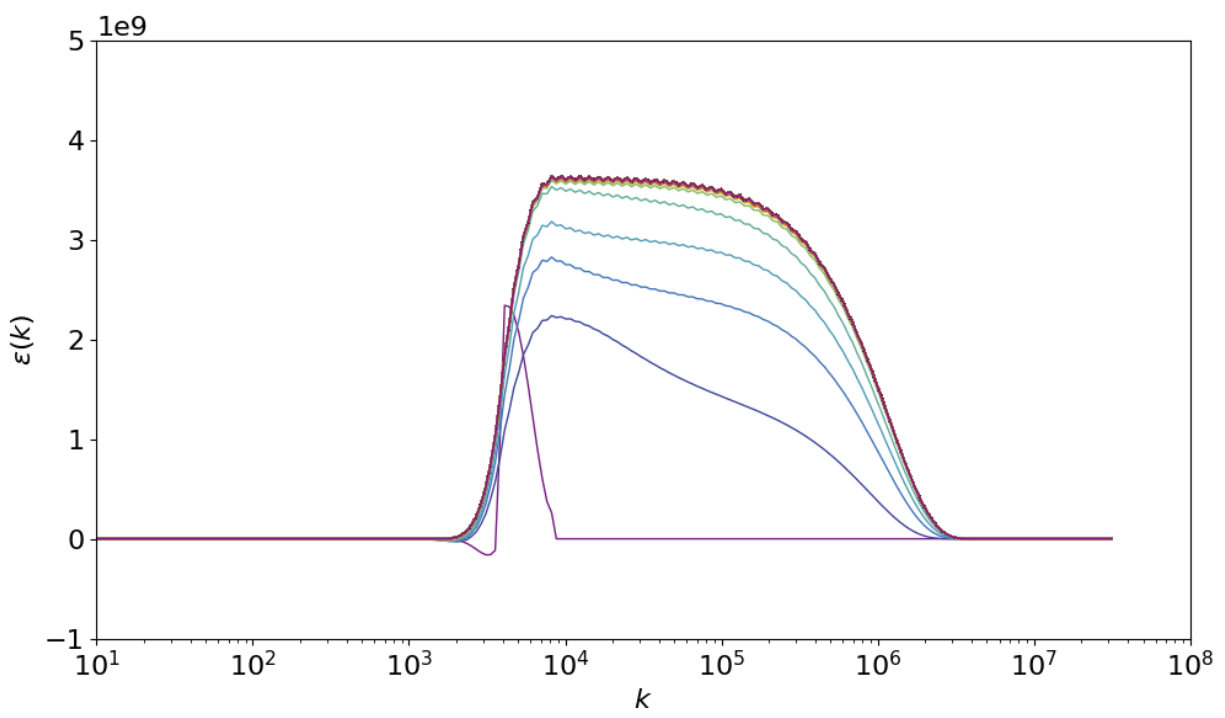

Figure 3. Time evolution of the spectral energy flux $\epsilon(k, t)$. The Kolmogorov $k^{-5 / 3}$ spectrum (see Fig. 2) corresponds to a positive flux while the large-scale $k^{2}$ scaling corresponds to a zero flux.

\section{Conclusions}

We have studied the focusing problem for the inviscid Leith model representing a propagation of the hydrodynamic turbulence spectrum $E(k, t)$ from a forcing scale towards small wave numbers $k$. In the Fourier space, this process corresponds to shrinking of an initial "hole" in the spectrum as time proceeds. This problem was 


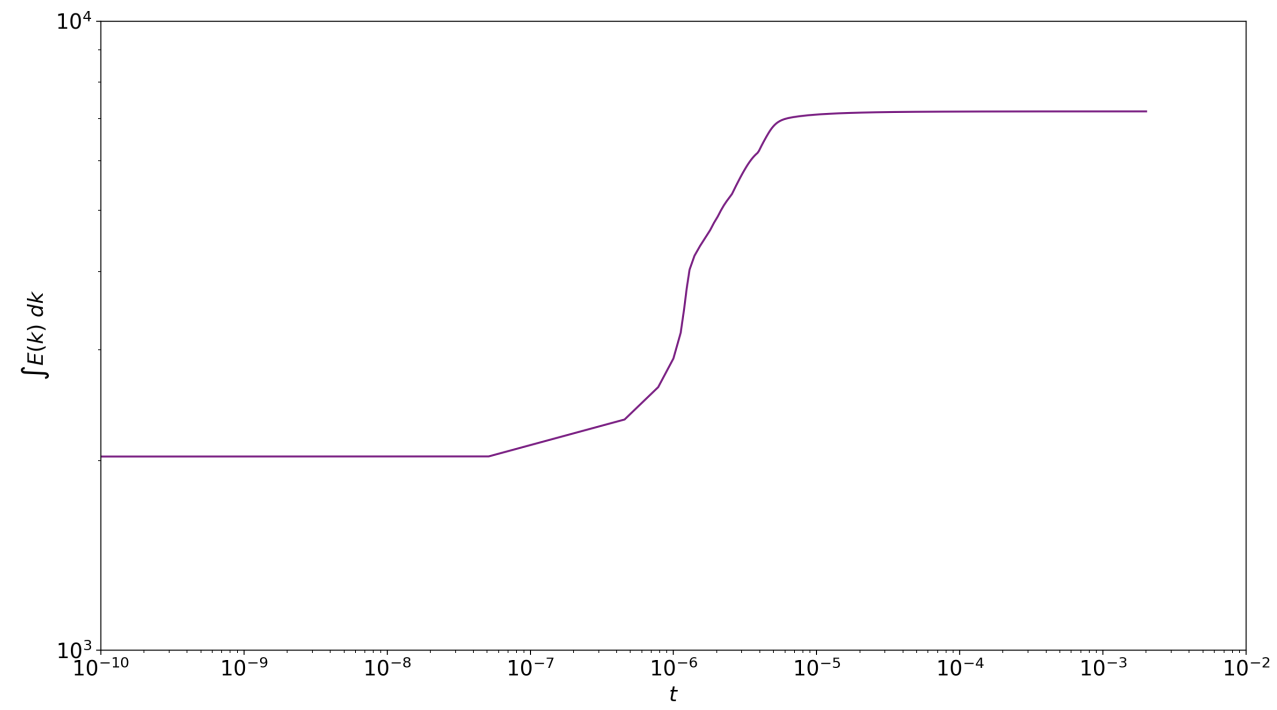

Figure 4. Time evolution of the energy $E(t)=\int E(k, t) d k$.

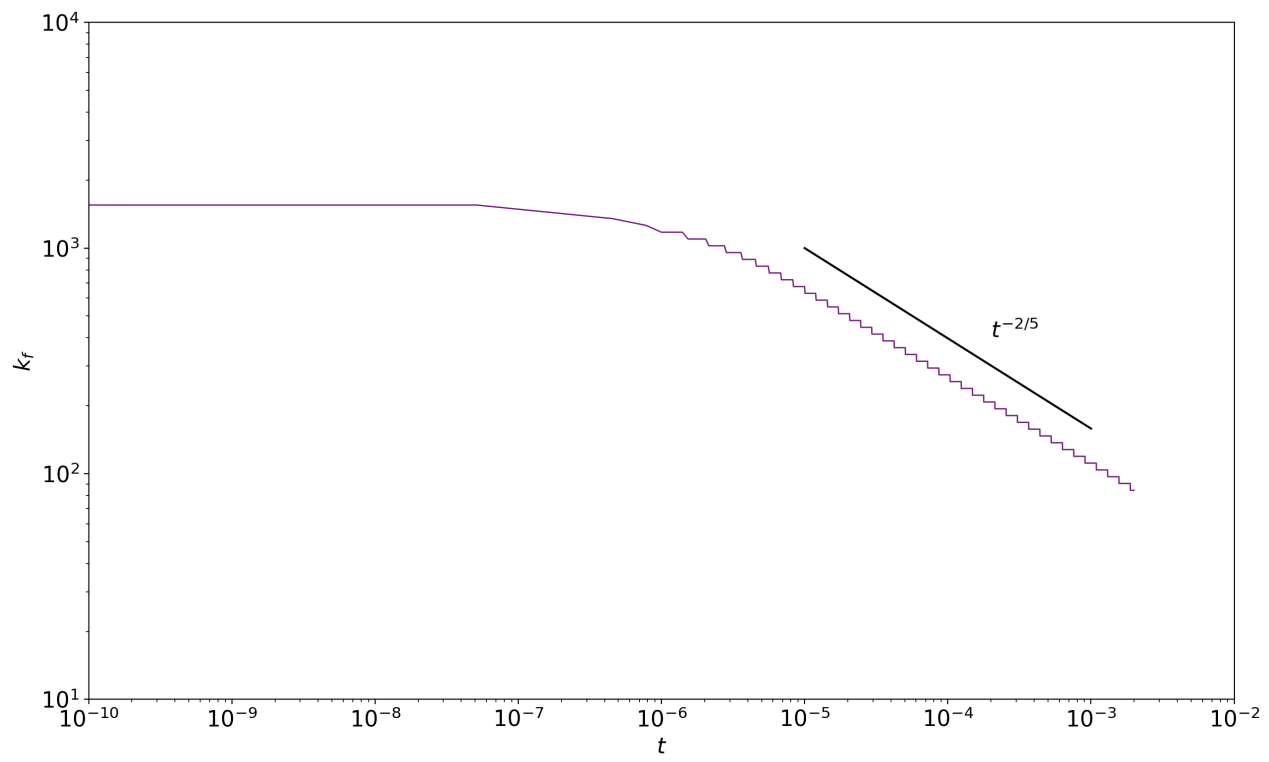

Figure 5. Time evolution of $k_{f}(t)$.

considered in a self-similarity formulation. The second-kind self-similarity was shown to be broken: the correct setup must be built using self-similarity of the third kind for which the similarity coefficients are fixed by boundary conditions arising from a preceding or a subsequent self-similar stage. Specifically in the present case, the similarity coefficients are determined by the subsequent steady-state thermodynamic spectrum $E(k)=k^{2}$, which dictates the boundary condition $F(\eta) \rightarrow \eta^{2}$ at $\eta \rightarrow \infty$. This condition selects 
the Saffman scaling [14]. The condition at the left boundary, $\eta=1$, is that both the spectrum and the energy flux turn into zero: this corresponds to a shrinking hole. We show that the shrinking time is infinite, and that there is a unique self-similar solution of the focusing problem satisfying the specified boundary conditions.

Using numerical simulations of the forced-dissipated Leith model, we have confirmed stability of our analytical self-similar solution and the fact that it is reached asymptotically for large time.

Formation of the thermodynamic spectrum $E(k)=k^{2}$ upscale of the forcing scale is a rather universal phenomenon common for most reasonable turbulence models. It was also observed in direct numerical simulations (DNS) of the forced Navier-Stokes equations in [15], the authors of which interpreted the result in terms of equivalence of the statistics upscale of the forcing to the equilibrium statistics in a truncated Euler equation. However, the details of the evolution towards such an equilibrium state have not yet been studied using DNS of the Navier-Stokes equations, and such a study would be an interesting subject for future research.

\section{Acknowledgements}

Sergey Nazarenko is supported by the Chaire D'Excellence IDEX (Initiative of Excellence) awarded by Université de la Côte d'Azur, France. Vladimir Grebenev's work was partially supported by the fédération Doeblin FR 2800, Université de la Côte d'Azur, France (code 180089013 01682).

\section{Bibliography}

[1] Leith C 1967 Diffusion Approximation to Inertial Energy Transfer in Isotropic Turbulence Phys. Fluids 101409 http://dx.doi.org/10.1063/1.1762300

[2] Grebenev V N, Nazarenko S V, Medvedev S B, Schwab I V and Chirkunov Y A 2014 Self-similar solution in Leith model of turbulence: anomalous power law and asymptotic analysis J. Phys. A: Math. Theor. 472025501

[3] Chirkunov Y A, Nazarenko S V, Medvedev S B and Grebenev V N 2014 Invariant solutions for the nonlinear diffusion model of turbulence J. Phys. A: Math. Theor. 4718185501

[4] Grebenev V N, Griffin A, Medvedev S B and Nazarenko S V 2016 Steady states in Leith's model of turbulence Phys A: Math. Theor. 4936365501

[5] Nazarenko S V and Grebenev V N 2017 Self-similar formation of the Kolmogorov spectrum in the Leith model of turbulence J. Phys. A: Math. Theor. 503035501

[6] Connaughton C and Nazarenko S 2004 Warm cascade and anomalous scaling in a diffusion model of turbulence Phys. Rev. Letters 924 044501-506

[7] Connaughton C and Nazarenko S 2004 A model equation for turbulence, arXiv:physics/0304044

[8] Clark T T, Rubinstein R and Weinstock J 2009 Reassessment of the classical turbulence closures: the Leith diffusion model J. Turbulence 10 1-23

[9] Zeldovich Ya B and Raizer Yu P 1966 Physiscs of Shock-waves and High-Temperature Phenomena vol 2 (Academic Press) p 157

[10] Galtier S, Nazarenko S V, Newell A C and Pouquet A 2000 A weak turbulence theory for incompressible MHD J. Plasma Phys. 63 447-488

[11] Nazarenko S V Wave turbulence 2011 (Berlin: Springer) 
[12] Cichowlas C, Bonaiti P, Debbasch F and Brachet M 2005 Effective dissipation and turbulence in spectrally truncated Euler flows Phys. Rev. Lett. 9526264502

[13] Vázquez J L 2007 The Porous Medium Equation. Mathematical Theory (Oxford: Oxford Science Publications) p 624

[14] Saffman P G 1967 Note on Decay of Homogeneous Turbulence Phys. Fluids 10 1349-1349

[15] Dallas V, Fauve S and Alexakis A 2015 Statistical equilibria of large scales in dissipative hydrodynamic turbulence, Phys. Rev. Lett. 11520204501

[16] Galtier S, Nazarenko S V, Buchlin E and Thalabard S 2019 Nonlinear diffusion models for gravitational wave turbulence Physica $D$ in press; arXiv:1809.07623v1 